INPLASY

PROTOCOL

To cite: Passos et al.

Wearables and Internet of Things (loT) Technologies for

Fitness Assessment: A

Systematic Review. Inplasy

protocol 202160041. doi:

10.37766/inplasy2021.6.0041

Received: 13 June 2021

Published: 13 June 2021

Corresponding author:

Sérgio Ivan Lopes

sil@estg.ipvc.pt

Author Affiliation:

Instituto Politécnico de Viana

do Castelo.

Support: ERDF.

Review Stage at time of this submission: Data analysis.

Conflicts of interest:

None declared.

\section{Wearables and Internet of Things (IoT) Technologies for Fitness Assessment: A Systematic Review}

Passos, J1; Lopes, Sl2; Clemente, FM33; Moreira, $\mathrm{PM}^{4}$; Rico-González, M5; Bezerra, P6; Rodrigues, LP7.

Review question / Objective: This work aims to identify and summarize recent studies that have used wearables and IoT technologies and discuss its applicability for fitness assessment.

Eligibility criteria: Inclusion Criteria: - Applications of wearable and IoT in fitness assessment (i.e., assessment of cardiorespiratory level, neuromuscular status, balance, sprint and change-of-direction, body mass or body composition) and health monitoring in athletes or sports (e.g., hearth rate, sleep quality); - Only original and full-text studies written in English; Exclusion Criteria: - Applications of wearables and loT in other human activities not related to fitness assessment or health monitoring in athletes (e.g., healthcare monitoring, well-being monitoring not related with sports, clinical populations, medical devices; - Written in other language than English. Other articles types than original (e.g., reviews, letters to editors, trials registrations, proposals for protocols, editorials, book chapters and conference abstracts); - The studies must specify the hardware and software of the wearable/loT device and include the adopted fitness assessment method.

INPLASY registration number: This protocol was registered with the International Platform of Registered Systematic Review and Meta-Analysis Protocols (INPLASY) on 13 June 2021 and was last updated on 13 June 2021 (registration number INPLASY202160041).

\section{INTRODUCTION}

Review question / Objective: This work aims to identify and summarize recent studies that have used wearables and IoT technologies and discuss its applicability for fitness assessment.

Rationale: Wearable and Internet of Things (IoT) technologies in sports open a new era in athlete's training, not only for 
performance monitoring and evaluation but also for fitness assessment. These technologies rely on sensor systems that collect, process and transmit relevant data, such as biomarkers and/or other performance indicators that are crucial to evaluate the evolution of the athlete's condition, and therefore potentiate its performance.

Condition being studied: Not applicable.

\section{METHODS}

Search strategy: Electronic databases (FECYD (WOS, CCC, DIIDW, KJD, MEDLINE, RSCI, SCIELO) IEEEXplore, PubMed, SPORTDiscus, Cochrane, and Web of Science) were searched for relevant publications prior to March 9, 2021. Keywords and synonyms were entered in various combinations in the title, abstract or keywords: (sport OR exercise OR "physical activity") AND ("wireless body sensor network" OR WBSN OR smartwatch* $^{*}$ OR watch OR clothing OR tracker* OR footwear OR wearable* OR "inertial measurement unit" OR IMU OR MEMS OR "microelectromechanical" OR accelerometer OR gyroscope OR barometer) AND (IOT OR "Internet of things") AND (performance OR movement* OR behavior* OR fitness OR cardio* OR "aerobic* " OR "strength" OR "neuromuscular" OR sprint* OR agility OR "change-of-direction" OR "heart rate" OR HR). Additionally, the reference lists of the studies retrieved were manually searched to identify potentially eligible studies not captured by the electronic searches. Finally, an external expert has been contacted in order to verify the final list of references included in this scoping review in order to understand if there was any study that was not detected through our research. Possible errata were searched for each included study.

Participant or population: Not applicable.

Intervention: Not applicable.

Comparator: Not applicable.
Study designs to be included: Not applicable.

Eligibility criteria: Inclusion Criteria: Applications of wearable and IoT in fitness assessment (i.e., assessment of cardiorespiratory level, neuromuscular status, balance, sprint and change-ofdirection, body mass or body composition) and health monitoring in athletes or sports (e.g., hearth rate, sleep quality); - Only original and full-text studies written in English; Exclusion Criteria: - Applications of wearables and IoT in other human activities not related to fitness assessment or health monitoring in athletes (e.g., healthcare monitoring, well-being monitoring not related with sports, clinical populations, medical devices; - Written in other language than English. Other articles types than original (e.g., reviews, letters to editors, trials registrations, proposals for protocols, editorials, book chapters and conference abstracts); - The studies must specify the hardware and software of the wearable/lot device and include the adopted fitness assessment method.

Information sources: Electronic databases (FECYD (WOS, CCC, DIIDW, KJD, MEDLINE, RSCI, SCIELO) IEEEXplore, PubMed, SPORTDiscus, Cochrane, and Web of Science).

Main outcome(s): This systematic review allowed us to analyze that internal and external load have been collected and analyzed separately. Future advances should add machine learning techniques to determine relationships between those variables and determine the optimum and individualized training targets for recreational and professional athletes, helping them to monitor and adjust the training process to the individual conditions and environmental factors.

Additional outcome(s): Not applicable.

Quality assessment / Risk of bias analysis: The majority of the works examined in this systematic have been conducted having in mind the application of Physiological Status Monitoring and Activity Recognition/ 
Tracking to fitness assessment. The focus of this systematic review is to evaluate the applicability of wearable and IOT technologies for such applications in terms of its hardware, software, and processing mechanisms, such as machine learning or other relevant tools. However, the current use of wearable and IoT technologies in sports to monitor athletes' internal and external workload is still in development.

Strategy of data synthesis: Not applicable.

Subgroup analysis: Not applicable.

Sensitivity analysis: Not applicable.

Language: English.

Country(ies) involved: Portugal.

Keywords: Wearables, Smart Wearables, IoT, IoT in Sports, Fitness Assessment.

Contributions of each author:

Author 1 - João Passos - Investigation and Original Draft Preparation.

Email: joao.passos@ipvc.pt

Author 2 - Sérgio Ivan Lopes Conceptualization, Investigation, Writing (Original Draft Preparation), and Supervision.

Email: sil@estg.ipvc.pt

Author 3 - Filipe Manuel Clemente Methodology, Investigation, Writing (Original Draft Preparation).

Email: filipe.clemente5@gmail.com

Author 4 - Pedro Miguel Moreira - Writing (Review \& Editing).

Email: pmoreira@estg.ipvc.pt

Author 5 - Markel Rico-González Methodology and Writing (Original Draft Preparation).

Email: markeluniv@gmail.com

Author 6 - Pedro Bezerra - Writing (Review \& Editing).

Email: pbezerra@esdl.ipvc.pt

Author 7 - Luis Paulo Rodrigues - Writing (Review \& Editing) and Project Administration.

Email: Iprodrigues@ese.ipvc.pt 